Review Article

\title{
A REVIEW ON APTAMERS IN GENE THERAPY AND THEIR APPLICATIONS
}

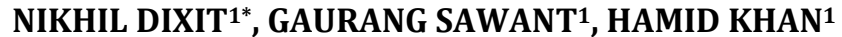 \\ 1Department of Pharmaceutics, H. K. College of Pharmacy, Oshiwara, Mumbai, India \\ Email: nikhild430@gmail.com
}

Received: 27 Jul 2020, Revised and Accepted: 17 Oct 2020

\begin{abstract}
Gene therapy has been initiated as long back from 1990s but is still in development. Research has been continuously going on in this field in order to cure genetic diseases by various techniques. One of these is aptamers. Aptamers are single stranded DNA or RNA molecules made by SELEX technology and have the ability of attaching to a variety of targets namely proteins, peptides, carbohydrates, toxins etc. They find application in skeletal disorders, biosensors, detection of viruses, delivery of drugs, various drug delivery systems, etc.
\end{abstract}

Keywords: Gene therapy, Cure, Aptamers, Skeletal, Biosensors, Viruses, Drugs

(C) 2020 The Authors. Published by Innovare Academic Sciences Pvt Ltd. This is an open access article under the CC BY license (http://creativecommons.org/licenses/by/4.0/) DOI: http://dx.doi.org/10.22159/ijpps.2020v12i12.39241. Journal homepage: https://innovareacademics.in/journals/index.php/ijpps.

\section{INTRODUCTION}

Humans have been suffering from diseases since ancient times, which includes external ones like Scabies, Ringworm, Chickenpox and so forth as well as internal ones like Influenza, Pneumonia, Acquired Immuno Deficiency Syndrome (AIDS) etc. Though treating some of them is not easy but most challenging to cure are disorders known as genetic disorders, which arise due to mutation in genes or defective genes with which a person is born with due to passing on the genes from either of the parents coupled with many environmental factors which also play a role in pathogenesis of the disorder. These disorders although scarce, have an impact on millions of people all over the world. Examples include Lesch Nyhan Syndrome, Down's syndrome, Cancer and Sickle cell anemia to name a few. In order to remedy such disorders, we require gene therapy. Gene therapy has been defined as introduction of nucleic acids into cells to treat, repair or replace and regulate defective genes to treat genetic diseases. The newly encoded nucleic acids will correct the faulty protein synthesis and the deficiency in the protein which was leading to the disease will be corrected [1]. There are many approaches towards this treatment like by employing antisense oligonucleotides, aptamers, liposomal gene delivery etc. Out of them, one such approach is by aptamers, which are defined as short single strand nucleic acid molecules, comprising of Deoxyribose Nucleic Acid (DNA) or Ribose Nucleic Acid (RNA) that attach to organic or inorganic molecules ranging from single atoms to an extensive variety of proteins. Aptamers are known by their high specificity to target molecule and binding power: dissociation constant of aptamer/target molecule complex comprising of repeated nanomolar units of RNA or
DNA bases is high so that they do not separate easily [2]. Due to the advancements in Systematic Evolution of Ligands by Exponential Enrichment (SELEX), which is now a fundamental technique for the separation of aptamers, multiple aptamers can be instantly selected in vitro against many targets, ranging from compact biomolecules to proteins and even on a cellular level [3]. The adoption technique of aptamers SELEX is an in vitro method. Briefly, SELEX is based on repetitive cycles of binding, splitting and multiplication of nucleotides. The primitive step of traditional SELEX is to cultivate the sequence pool with the target (protein, nucleic acid, etc.). The sequence pool comprises of nucleic acid library containing $10^{14}-10^{15}$ revisions of random 30-100 nucleotides surrounded by persistent sequences at both ends. The casual region accommodates the sequences that will be evaluated for top specificity and affinity to the target. Second step if that the sequences that attach successfully to the target are retained, while unbound nucleotides are released from the cycle. The third step is to cleanse and multiply the fused sequences to form a novel sequence pool for the next cycle. This cyclic process is usually repeated 8-15 times before attaining the appropriate aptamer sequence pool which is needed to complete the process [4, 5]. Literature survey was done using accessible databases such as Google scholar, PubMed, Scopus to combine research articles. The objective of this review is to provide all the literature on aptamers in the last $10 \mathrm{y}$ followed by their applications.

\section{Aptamers}

The structure of aptamer used therapeutically is given below as shown in fig. 1.

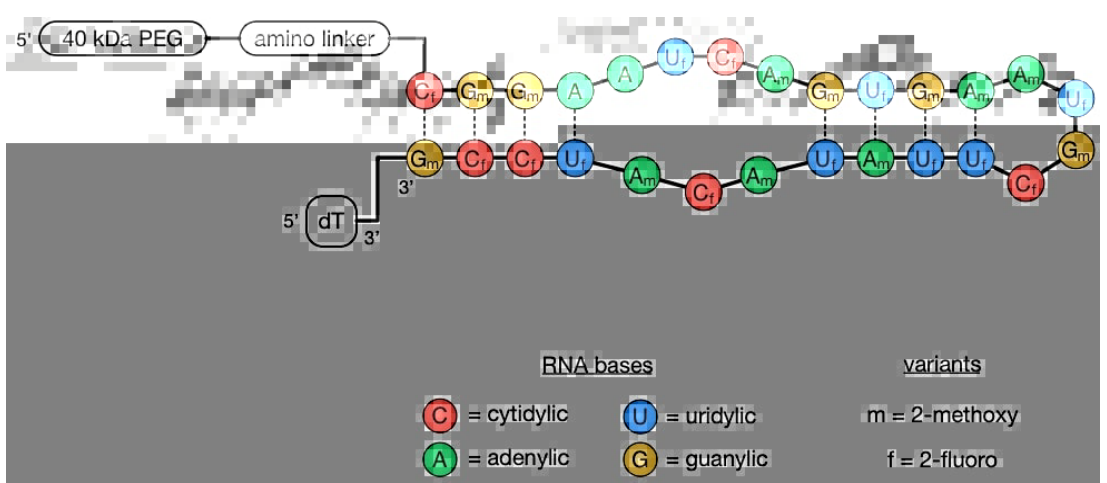

Fig. 1: Structure of aptamer pegaptanib (Macugen), the foremost nucleic acid aptamer to be given permission by the US FDA for clinical use [6] 


\section{Adapted by permission from the ATD Bio (Chemical industry in Southampton, England)}

Aptamers are novel target molecules for therapeutic intervention and diagnostic, aptamer technology has a great potential to become a source of lead compounds. For an assembly of aptamers there is no need any animals or specific cell lines; thus they can be pushed up against noxious molecules or non-immunogenic targets with apex rapport and specificity [7]. The characteristics of bacterial virus phi29 packaging RNA (pRNA) molecules were employed to unite it with a hexameric ring nanostructure of aptamer, thereby inclined towards delivering multiple therapeutics into specific cells [8]. Experiment involving adding different receptor-binding ligands to pRNA double-stranded $5^{\prime} / 3^{\prime}$ end helical domain resulted in chimeric molecules such as pRNA/aptamer [9]. DNA nanostructures can also be influenced to fabricate molecular machines of variable complexities. These molecular devices can be run to liberate many types of cargos at specific targets by conformational switching. This conformational change is induced by a molecular effector or by intermolecular hybridization with complementary nucleic acids. Aptamer-based biosensor technology is the energetically-growing aptamer field and pledges to continuously pop up with new clinical or twin drug diagnostic and personalized theranostic assays. A lot of academic laboratories exploit the capacity of aptamers to change their conformation upon ligand binding to develop label-free analytical assays [10]. A construct which is able to detect avian influenza virus reserved as aptasensor was developed. Quartz crystal microbalance (QCM) method was used, which includes attachment of quartz particles to the polymeric, porous hydrogel, containing DNA aptamers. Not long ago, the avian influenza was an epidemiological hurdle; the disease hereby designated by a severe course, high mortality rate and escalated risk of zoonotic influenza strain production. It has the ability of being transmitted from one person to person. Hemagglutinin (HA), a popular influenza protein typically a glycoprotein present in pinnacle amounts on the viral surface and is the cause for amalgamation of the virus with the host cell. There are a minimum of 18 variable HA antigens; therefore, they could serve not only for infection discovery in addition to differentiate between current influenza types and subtypes. Aptamers are able to decide between influenza types A from type B or even allied strains of the same influenza subtype. It should be noted that P30-10-16 lashes to the target molecule (H3N2 of virus A) with more than 15 -fold elevated affinity, as compared to standard anti-HA monoclonal antibody. Just now, aptamers were manufactured which are able to detect influenza virus type A: H1N1 and H3N2, along with avian virus, H5N1. It is still indispensable to make simple and widely available methods in order to further expand the benefits of aptamers. Another salient area of applications of aptamers is the detection of chronic infections caused by viruses of hepatitis B and C [11]. Aptamer specific for HCV E2 glycoprotein was unearthed, which brought into light a novel diagnostic testEnzyme-Linked Apto-Sorbent Assay (ELASA) - which permitted for qualitative analysis, in addition to quantification of virus particles in the assessed samples [12]. An important impact for expansion in viral diagnostics with aptamers have been made possible by constructing gold microelectrodes with impedimetric properties in order to decide between biologically active from inactive form of the virus and used heat-inactivated vaccine as a prototype. Using fixed set of DNA aptamers which were exposed, the presence of the viable virus form could be identified since the probability of error was lowered significantly [13]. Similar study was taken up, which in turn defined the target of constructed aptamer as a glycosylated HA available on infected cells. It is important to blossom the line of fast, quick responding, and economically diagnostic tools, to pinpoint the type of virus infection, as well as detection of dysplastic endometrial cells with the hazard of malignant metamorphosis. As a, HPV is a prospective target for aptamers [14]. An experiment done by making use of in vitro selection to obtain the RNA aptamer that tethers to viral E7 oncoprotein. Being a section of the biosensor it could equip a powerful diagnostic tool which helps to detect virus within the human body [15]. A fluorescent-conjugated DNA aptamer was developed that has the power to bind surface determinants of the normal cervical epithelium. The cells that started malignant transformation failed to fuse with the aptamer [16]. DNA aptamers were constructed, which were targeted as antagonists towards the pathogens of the arbovirus family, including a variety of viruses, such as Chikungunja, Crimean-Congo Hemorrhagic Fever, Dengue, West Nile and Tick-Borne Encephalitis. They got sequences with high precision and rapport for both the clean recombinant viral proteins and whole inactive viruses. They demonstrated the merit of chosen sequences in forecasted diagnostic methods, i.e., lateral flow chromatographic test strip and fluorescent aptamer-magnetic bead sandwich assay. They also brought forward the aptamers to be serviced for pliant immunity and antiviral prevention because of the shallow immunogenicity. This technique could be bright in any dangerous infection with shortfall of well-organized therapeutic replacement. The application of biosensors loaded with the aptamers or differing research techniques by adopting these molecules permits for sensing of both: early (genetic material, viral proteins) and late (host own antibodies) infection markers [17]. There is a high probability of infection and astonishing frequency of mutations, leading to a cyclical emergence of novel viral strains with epidemic or even pandemic danger as a result focused attention was given to influenza diagnosis. It is still necessary to generate simple and available technics in order to increase the benefits of aptamers [18]. To weaken HIV-associated cardiomyopathy an antiglycoprotein120 aptamer called as UCLA1 was established. It directly fuses to HIV-1 thereby destroying the virus, thus granting cardiomyocytes from cell death and indirectly halted infection of monocyte-derived macrophages in the heart [19].

\section{Diagnostic works with aptamer-based biosensors performed on} natural or clinical-based samples

The efficacy of aptasensors in the recognition of viruses was also evaluated in clinical samples. An experiment was undertaken to merge aptamers with biosensor technology, known as Surface Plasmon Resonance (SPR). This model incorporates a fragment coated with both gold coting and Streptavidin as the drug. DNA aptamers launched against avian influenza H5N1 have been fixed to its surface, since they were reconstructed by biotinylation; the material altered in the study was saliva collected from the poultry. The method has absence of labeling procedures and enables quick $(1.5 \mathrm{~h})$ detection [20]. In another study twin aptamers called as IF10 and IF22 were constructed, became attached at different sites of H5N1 virus, and playing a dual role of capturing probe as well as signaling probe, respectively. This resulted in a sandwich-type SPR biosensor platform for the sensitive detection of H5N1 viruses. The aptasensor, consisted of $\mathrm{H} 5 \mathrm{~N} 1$ virus attached to a biotin-labeled aptamer IF10, which was strapped onto the surface of the streptavidin-coated SPR gold chips. The detection ability shown by this aptamer was comparable to that of Enzyme-linked immunosorbent assay (ELISA) [21]. A solitary-molecule real-time aptasensor for recognizing HIV-1 was constructed. This study employed a combination of Nano pores, resistive-pulse technique, and an RNA aptamer which is specific towards the HIV-1 nucleocapsid protein 7 known as SL3. A voltage was passed across a silicon nitride membrane, and the strength of the ionic current flowing through the nano-pores on the membrane was assessed. The passage of aptamer-protein complex through the membrane, interrupted the current, which was in turn restored by a translocation event signal thus confirming the presence of the protein and subsequent detection of the virus [22].

\section{Pros and cons of aptamer-based tests in comparison to other diagnostics methods}

The ongoing diagnostic methods for detection of viral infections are mostly ELISA or molecular biology tests, which are routinely employed $[20,23,11,14]$. Repeatedly used ELISA is a multi-stage method and is less efficient due to the fact of relatively shallow sensitivity as well as high incidences of false-positive results $[20,23,11]$. As a substitute regularly used or even investigational diagnostic molecular tests permit for quickest detection of the new genetic material, without standing by for the immune reaction of the host. Furthermore, the supremacy of molecular assays is their very high sensitivity: they have the ability to catch lone viral copies and/or early viral transcripts right away after the genesis of infection [25, 26]. But these have their shortcomings such as not economically feasible, 
complex procedures and requiring trained persons, as well as they, are hardly ever used in practice. Aptamers seem to be an appropriate solutions to the hindrances described above. They show light as a stunning alternative to the traditionally used procedures, due to their high specificity, power of binding to whichever comes to hand viral antigen and low cost of manufacture. The application of biosensors armed with the aptamers or additional research methods using these molecules permits for detection of both: newly manifested (genetic material, viral proteins) and delayed (host own antibodies) infection markers [23, 24]. Also, aptamers allow to choose between infected host cells and not infected ones and maybe supportive to point out active incarnations of the virus $[13,16]$ Table 1 compares a few of the traditional diagnostic techniques employed in the recognition of influenza, HBV and HIV as given below. It is worthwhile to note that in many scenarios, the use of currently available diagnostic tools has a number of limitations, which can be bypassed using aptamers $[27,28]$. Moreover, the aptamerbased minimum boundary of detection is occasionally lesser than in RT-PCR, in the case of influenza virus [11, 27]. In contrast, when the detection threshold is indistinguishable, or even more as compare to modern used techniques, and aptamers guarantee enhanced sensitivity as well as specificity of the diagnostic assay [20].

Table 1: Comparison of traditionally used diagnostic techniques in the detection of HIV, HBV and influenza

\begin{tabular}{llllll}
\hline Virus & Method & Detection limit & Advantages & Disadvantages & References \\
\hline Influenza & RT-PCR & $0.0256 \mathrm{HAU} / \mathrm{ml}$ & Specificity & Expensive & Dhumpa R et al.2011 [27]. \\
& qRT-PCR & $1.785 \mathrm{IU} / \mathrm{ml}$ & Sensitivity & Complicated, highly skilled stuff & Sidoti F et al. 2010 [28]. \\
HBV & ELISA & $0.5 \mathrm{pg} / \mathrm{ml}$ & Specificity & Expensive & Yang J et al. 2010 [29]. \\
& qRT-PCR & $18 \mathrm{IU} / \mathrm{ml}$ & Sensitivity & Complicated, highly skilled stuff & Kim H et al. 2013 [30]. \\
HIV & ELISA & $0.9-1.2 \mathrm{IU} / \mathrm{ml}$ & Specificity & Expensive & Mühlbacher A et al. 2013 [31]. \\
& qPCR & $3.21-11.60 \mathrm{IU} / \mathrm{ml}$ & Sensitivity & Complicated, highly skilled stuff & Saune K et al. 2013 [32]. \\
\hline
\end{tabular}

\section{Applications of aptamers}

\section{Aptamers in bio imaging}

One application of aptamers is in bio-imaging, where employing an aptamer that is fused to a fluorophore, a quantum dot (QD) or other materials such as gadolinium which is functional for magnetic resonance imaging (MRI). Using aptamers as imaging agents has the superiority of being non-toxic due to the fact oligonucleotide moieties exist in the human body. Results were published of glioma cell line (C6) cell imaging using a dye called as Cyanine 3(Сy3) which was labeled onto AS1411 aptamer, which had undergone chemical modification of 5-( $N$-benzylcarboxyamide $)-2{ }^{\prime}$-deoxyuridine (5'BzdU) on a thymidine base. The AS1411 aptamer is particular for the nucleolin trans-membrane protein present in cancer cells. Specifically, this group enhanced the aptamer's binding power to its target via a chemical modification. The cell imaging of the altered Cy3-labeled AS1411 aptamer was more systematic for the C3 cells as compared to the original Cy3-labeled AS1411 aptamer [33]. Assembly of QD-A10 and DUP-1 aptamer complex was done, which is particular for Prostate-specific membrane antigen [PSMA (+)] and [PSMA (-)] prostate cancer cells (LNCaP and PC3), was demonstrated by imaging to attach only to prostate cancer cells instead of normal (PNT2) or other cancer cells [34].

\section{Aptamers in delivery of anthracycline drugs and imaging}

The majority of theranostic studies with aptamers involve the codelivery of imaging agents in addition to anthracycline drugs (predominantly Doxorubicin). These drugs deal with DNA by intercalation, halting replication, and disrupting gene expression. The first way to administer the drugs was to entrap them in different types of nanoparticles that were further labeled for recognition by imaging by attaching to aptamers. This method was used where doxorubicin in mesoporous silica nanoparticles that were further bonded with an anti-MUC1 aptamer and radiolabeled by [99] Technetium. Fixed targeting in addition to drug delivery of the nanoparticle was exhibited in vitro on cells and scintigraphy demonstrated that the nanoparticles were able to choose a subcutaneous tumor in vivo [35]. A similar strategy was used where in the loading of superparamagnetic iron oxide nanoparticles (SPIONs) and Doxorubicin in poly (lactic-co-glycolic acid) (PLGA)based nanoparticles were done which were further joined to the anti-nucleolin aptamer. Higher tumor targeting was achieved by MRI for the nanoparticles bonded with the aptamer than for non-bonded nanoparticles. Additionally, the nanoparticles bonded with the aptamer gave much higher inhibition of tumor growth and ensured the sustained life span of mice having C26 colon carcinoma xenografts $[36,37]$. Yet another procedure to administer drugs is to permanently attach them to the surface of nanoparticles. This strategy was employed to bond an anti-MUC1 aptamer to quantum dots and further joined Doxorubicin to their surface via a $\mathrm{pH}_{-}$ sensitive hydrazone bond. These bonds do not break down in blood at a neutral as well as slightly basic $\mathrm{pH}$ but undergo fast hydrolysis in the acidic surrounding of endosomes. As a result it should free the drug only in cancer cells that have nucleolin because they should incorporate the nanoparticles via the aptamer. In vivo fluorescence imaging showed that the quantum dots amassed more in subcutaneous xenografts when they were joined to the aptamer. Furthermore, the therapeutic response of these nanoparticles were enhanced marginally by the aptamer in vitro, but was not assessed in vivo due to some issues [38]. Doxorubicin was also joined to the surface of shining gold nano-clusters which was further bonded with two specific targeting moieties: the anti-nucleolin aptamer and a cyclic Arg-Gly-Asp (cRGD) peptide, which joins to $\alpha v \beta 3$ and $\alpha v \beta 5$ integrins, which are overexpressed within tumor endothelial cells. In vivo fluorescence imaging showed considerable higher tumor targeting when the nanoparticles were joined with the dual targeting agents than when they were lonely and somewhat greater tumor-targeting than nanoparticles bonded with the only cRGD. Intravenous injection of the nanoparticles bonded with the dual targeting agents every $48 \mathrm{~h}$ generated higher retardation of tumor growth than non-bonded nanoparticles and also on Doxorubicin alone [39]. An aptamer that bonds to the prostate-specific membrane antigen (PSMA), which is overexpressed on several prostate tumors was developed. The extension the sequence of the aptamer by an auxiliary (CGA) 7 repeat that was inter-mixed to a sequence conjugated earlier to the surface of SPIONs was performed. This dual strand matching was used to conjugate the aptamer as well as for the entrapment of Doxorubicin, which is known to preferably attach to following cytosine and guanine base pair regions. The biodistribution of this complex was then checked by MRI in nude mice showing subcutaneous tumors. Following an hour after administration the nanoparticles bonded with the aptamer showed ten-fold greater tumor-targeting than those joined with a scrambled control sequence [40]. A similar loading strategy employed which involved entrapment of a MUC1 aptamer that was joined to SPIONs, with Epirubicin. MRI demonstrated higher tumor targeting of the nanoparticles conjugated with the aptamer than unconjugated nanoparticles, similarly to the previous study. Furthermore, they also demonstrated greater retardation of tumor growth [41]. The ability of Doxorubicin to intercalate into DNA was also exploited to construct an intelligent split aptamer-based activatable theranostic probe. They employed the Sgc8c aptamer that deals with a cancer-related membrane PTK-7. The Sgc8c aptamer was broken into two parts and joined via a short DNA linker labeled with a fluorophore. The central part of this linker was then inter-mixed with a complementary short DNA strand to which was linked to a quencher that diminishes the fluorescence emission of the fluorophore. It was also armed with Doxorubicin, as the double helix region linker/short DNA strand was guanine and cytosine rich. The model was designed to be pulled apart when the 
structure of the aptamer encounters metamorphosis during its interaction with PTK-7, delivering the Doxorubicin and activating the radiation of a fluorescent signal. Both therapy as well as imaging, were tested in vitro. The checking of the construct in vivo by fluorescence imaging in nude mice bearing subcutaneous tumors was also carried out. The tumor demonstrated high fluorescence, just 15 min post-injection, but the clinical response was not evaluated [42].

\section{Aptamers in delivery of taxane drugs and imaging}

An overview of aptamers employed in various drug delivery systems, including cancer and other diseases, is shown in fig. 2 .

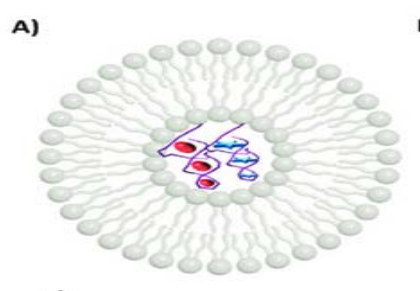

D)
B)

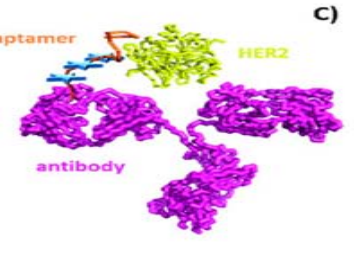

C)

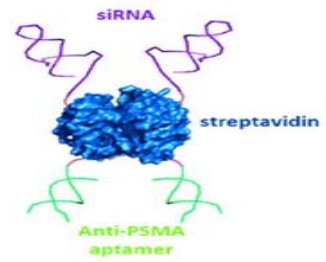

E)
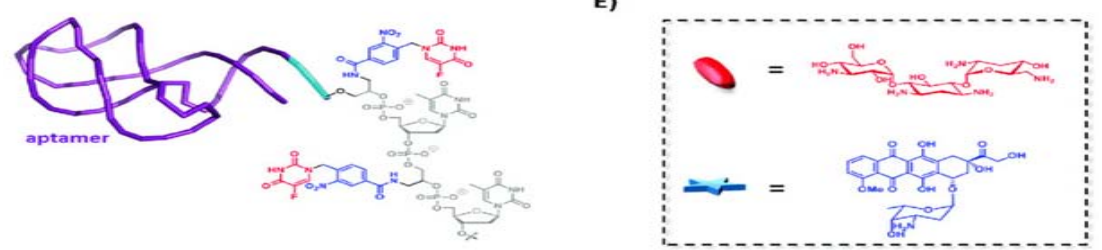

Fig. 2: An overview of various aptameric systems used in drug delivery [43], A] Entrapment of aptamer drug complex in liposome, B] Aptamer antibody conjugate for targeting selective genes, C] Drug streptavidin serving as a base for connecting two aptamers and two siRNA molecules, D] Drug 5-flourouracil shown in red connected to the aptamer oligonucleotide backbone via a photocleaveable linker which is shown in blue, E] Chemical structures of Tobramycin (red) and Doxycycline (blue)

Copyright permission obtained under creative commons (CC BY) license (http://creativecommons.org/licenses/by/4.0/)

Several theranostic studies have been conducted with aptamers for the co-delivery of imaging agents and taxane drugs, such as Paclitaxel (Taxol) and Docetaxel (Taxotere), which are commonly employed as chemotherapy agents. The main mechanism of action of these drugs is to wreak havoc upon the microtubule duty that is pivotal for cell division. Opposite to anthracyclines, which are watersoluble, taxanes are water-insoluble. Thus, the theranostics nanosystems were designed for those drugs which are not alike. A method was established using a nano-precipitation process with an acetone/water system to build nanoparticles of cholic acid-derived with a star-shaped block copolymer comprising of polylactic glycolic acid (TPGS) and Vitamin E armed with Docetaxel and a near-infrared fluorescent dye. The surface of these nanoparticles was moreover linked with the anti-nucleolin aptamer. The constructed nanoparticles were checked in two mouse models wherein the mice obtained subcutaneous tumor xenografts from human breast cancer cell lines. The nanoparticles linked with the aptamer showed fourfold higher tumor-targeting $24 \mathrm{~h}$ following after intravenous injection than non-bonded nanoparticles or free dye by fluorescence imaging. Furthermore, they also illustrated higher inhibition of tumor growth [44]. The anti-nucleolin aptamer was also exploited again to target poly (L-c-glutamyl-glutamine) nano-conjugates armed with Paclitaxel as well as a fluorescent dye. In contrast to most of the studies described above, these nanoparticles were not checked in subcutaneous models but in nude mice that institute orthotropic brain tumors from an intracranial injection of human U87 MG glioblastoma cells. Fluorescence imaging showed two-fold higher tumor targeting by the nanoparticles linked with the aptamer than lone nanoparticles $24 \mathrm{~h}$ following intravenous injection. Additionally, when the nanoparticles were administered every three days, the median life span of mice that obtained the nanoparticles (52 d) was substantially prolonged as compared to those treated with unconjugated nanoparticles (47 d), free Taxol (40 d), or a control saline solution (36 d) [45]. Not so different conclusions were seen in an alternative orthotropic brain tumor model. The also similarly employed the anti-nucleolin aptamer joined to the surface of poly (ethylene glycol)-poly ( $\varepsilon$-caprolactone) nanoparticles armed with Docetaxel and fluorescence dye. The surface of the nanoparticle was also joined with a peptide earlier protected by phage display to travel across the Blood-Brain Barrier (BBB). Fluorescence imaging illustrated that this peptide improved brain ingestion of the nanoparticles as well as the addition of the aptamer, further enhanced ingestion of the nanoparticles by the tumor [46]. The same nanoparticle was used, which in turn attained the same result using an alternative aptamer chosen to recognize glioblastoma cancer cells in the absence of the peptide to travel across the BBB. This may be accountable to the truth that BBB is imaginarily hampered throughout the development of the brain tumor [47].

\section{Aptamers in miscellaneous applications}

Hydrogels are inter-linked polymers comprising of a 3 Dimensional (3D) network. It is true that hydrophilic group is often present in chemical structures of many compounds, including hydrogels contain groups such as carboxylic acid $(\mathrm{COOH})$, imine $(\mathrm{NH})$, hydroxyl $(\mathrm{OH})$, sulphonic acid $\left(\mathrm{SO}_{3} \mathrm{H}\right)$ and carboxamide $(\mathrm{CONH})$, hydrogels also possess the capability to hold and absorb a fixed amount of water which leads to their swelling when they come in contact with water thus forming the 3D network [48]. Aptamer-derived hydrogels can be scheduled to deliver many and multiple therapeutics when required through particular nucleic acid identification and complementary inter-mixing process. Recently a nucleic acid-based affinity hydrogel system for controlled protein release was assembled. Two variable nucleic acid aptamers, as well as complementary sequences, were employed to control the delivery speed of Vascular Endothelial Growth Factor (VEGF) and plateletderived growth factor having two B subunits (BB) in vitro [49-51]. A novel aptamer-functionalized hydrogel for controlled cell capture and liberation was fabricated. The customizable hydrogel comprises of a chief Complementary Sequence (CS), which is an aptamer sequence and a subsidiary CS. Firstly, the main CS was linked to the hydrogel resting on a solid support surface. The aptamer was intermixed with the key CS and can only bind bond with cells via polyvalent aptamer-protein interactions. When the secondary CS was applied to trigger the hydrogel, the aptamer broken apart from the main CS and then inter-mixed with the subsidiary CS. Therefore, polyvalent interactions between the cells and the hydrogel were diminished, resulting in a safe release of the cells from the solid surface [52]. An aptamer-derived hydrogel as a synthetic Extracellular Matrix (ECM) for cell fixing without compromising cell viability was fabricated [53]. 


\section{Aptamers for skeletal disease therapy in preclinical studies}

Stem-cell-based therapies are one of the most innovative and rewarding fields of making advancements in the treatment of diseases in humans [54]. Stem cells are those cells which are in their premature state and have not yet been differentiated to handle a specific task. These cells are also immortal owing to their inability to age over time coupled with the ability to keep multiplying forever and such cells have the power to transform to variable cell types like skin, liver, kidney, heart, nerve cells and so forth depending upon the type of organ in which they are introduced [55]. The cell count of bone marrow mesenchymal stem cells (BMSCs) is diminished through aging while enhanced through adipocyte polarity. It is already demonstrated that miR-188 level is much greater in BMSCs in old as compared to young mice and human. Animals deficient in miR-188 can be safeguarded from age-related bone loss and fat collection in bone marrow. An aptamer that only recognizes BMSCs is constructed and linked with miR-188 to form a nano complex was constructed. This targeted transported nano-complex could enhance bone generation and lessen fat collection in bone marrow with more clinical results in aged mice, showing a promising approach for agerelated bone loss cure [56]. Another preliminary study gave birth to a windowed aptamer targeted against human jaw periosteal cells (JPCs) is constructed for tissue engineering in oral and maxillofacial surgery. This aptamer has great power to bind to human osteogenically generated JPCs and BMSCs from bone marrow while it shows an absence of binding to any other cell lines or undifferentiated JPCs or JPCs born from alternative sources. It can be used to cleanse osteogenic parent cells from undifferentiated JPCs or stem cells from another sources. The mineralization ability is greater in the positive aptamer group, which is an encouraging method for tissue engineering [57].

\section{CONCLUSION}

In order to materialize a proper therapy for genetic diseases it is necessary to understand what those diseases are and how they can be treated using methods such as aptamers. The literature work done in aptamers and subsequently its applications were discussed. This article will definitely help all researchers, students, as well as academicians who are working in this field. Even now advancements are currently going on in this field and in the future further developments will ensure that genetic diseases are cured successfully and people who are suffering from this disease will lead normal lives.

\section{ACKNOWLEDGMENT}

The authors are thankful towards Mrs. Faiza Shaikh for providing guidance during the preparation of this manuscript.

\section{FUNDING}

Nil

\section{AUTHORS CONTRIBUTIONS}

All authors have taken part in the design and drafting the article and revising it critically for important intellectual content as well as approval of the final version.

\section{CONFLICT OF INTERESTS}

\section{Declared none}

\section{REFERENCES}

1. Hardee CL, Arevalo Soliz LM, Hornstein BD, Zechiedrich L. Advances in non-viral DNA vectors for gene therapy. Genes 2017;8:65.

2. Zhou J, Bobbin M, Burnett JC, Rossi JJ. Current progress of RNA aptamer-based therapeutics. Front Genet 2012;3:234.

3. Han K, Liang Z, Zhou N. Design strategies for aptamer-based biosensors. Sensors 2010;10:4541-57.

4. Davydova A, Vorobjeva M, Pyshnyi D, Altman S, Vlassov V. Aptamers against pathogenic microorganisms. Crit Rev Microbiol 2016;42:847-65.

5. Torres Chavolla E, Alocilja EC. Aptasensors for detection of microbial and viral pathogens. Biosens Bioelectron 2009;24:3175-82.
6. Tom Brown, Dr Tom Brown. Nucleic Acids Book, free online book on the chemistry and biology of nucleic acids. Available from: https://www.atdbio.com/content/61/Nucleic-acidaptamers [Last accessed on 20 May 2020].

7. Rozenblum GT, Lopez VG, Vitullo AD, Radrizzani M. Aptamers: current challenges and future prospects. Expert Opin Drug Discovery 2016;11:127-35.

8. Shu Y, Cinier M, Shu D, Guo P. Assembly of multifunctional phi29 pRNA nanoparticles for specific delivery of siRNA and other therapeutics to targeted cells. Methods 2011;54:204-14.

9. Zhou J, Shu Y, Guo P, Smith DD, Rossi JJ. Dual functional RNA nanoparticles containing phi29 motor pRNA and anti-gp120 aptamer for cell-type specific delivery and HIV-1 inhibition. Methods 2011;54:284-94.

10. Seok Kim Y, Ahmad Raston NH, Bock Gu M. Aptamer-based nanobiosensors. Biosens Bioelectron 2016;76:2-19.

11. Wang R, Li Y. Hydrogel based QCM aptasensor for detection of avian influenza virus. Biosens Bioelectron 2013;42:148-55.

12. Park JH, Jee MH, Kwon OS, Keum SJ, Jang SK. Infectivity of hepatitis C virus correlates with the amount of envelope protein E2: development of a new aptamer-based assay system suitable for measuring the infectious titer of HCV. Virology 2013;439:13-22.

13. Labib M, Zamay AS, Muharemagic D, Chechik AV, Bell JC. Aptamer-based viability impedimetric sensor for viruses. Anal Chem 2012;84:1813-6.

14. Parekh P, Tang Z, Turner PC, Moyer RW, Tan W. Aptamers recognizing glycosylated hemagglutinin expressed on the surface of vaccinia virus-infected cells. Anal Chem 2010;82:8642-9.

15. Toscano Garibay JD, Benítez Hess ML, Alvarez Salas LM. Isolation and characterization of an RNA aptamer for the HPV16 E7 oncoprotein. Arch Med Res 2011;42:88-96.

16. Graham JC, Zarbl H. Use of cell-SELEX to generate DNA aptamers as molecular probes of HPV-associated cervical cancer cells. PLoS One 2012;7:e36103.

17. Bruno JG, Carrillo MP, Richarte AM, Phillips T, Andrews C. Development, screening, and analysis of DNA aptamer libraries potentially useful for diagnosis and passive immunity of arboviruses. BMC Res Notes 2012;5:e633.

18. Wandtke T, Wozniak J, Kopinski P. Aptamers in diagnostics and treatment of viral infections. Viruses 2015;7:751-80.

19. Lopes de Campos WR, Chirwa N, London G, Rotherham LS, Morris L. HIV-1 subtype C unproductively infects human cardiomyocytes in vitro and induces apoptosis mitigated by an anti-Gp120 aptamer. PLoS One 2014;9:e110930.

20. Bai H, Wang R, Hargis B, Lu H, Li Y. A SPR aptasensor for detection of avian influenza virus H5N1. Sensors 2012;12:12506-18.

21. Nguyen VT, Seo HB, Kim BC, Kim SK, Song CS. Highly sensitive sandwich-type SPR based detection of whole H5Nx viruses using a pair of aptamers. Biosens Bioelectron 2016;86:293-300.

22. Niedzwiecki DJ, Iyer R, Borer PN, Movileanu L. Sampling a biomarker of the human immunodeficiency virus across a synthetic nanopore. Acs Nano 2013;7:3341-50.

23. Fletcher SJ, Phillips LW, Milligan AS, Rodda SJ. Toward specific detection of dengue virus serotypes using a novel modular biosensor. Biosens Bioelectron 2010;26:1696-700.

24. Ruslinda AR, Tanabe K, Ibori S, Wang X, Kawarada H. Effects of diamond-FET-based RNA aptamer sensing for detection of the real sample of HIV-1 tat protein. Biosens Bioelectron 2013;40:277-82.

25. Ocadiz Delgado R, Albino Sanchez ME, Garcia Villa E, Aguilar Gonzalez MG, Cabello C. In situ molecular identification of the Influenza a (H1N1) 2009 neuraminidase in patients with severe and fatal infections during a pandemic in Mexico City. BMC Infect Dis 2013;13:20.

26. Caliendo AM. Multiplex PCR and emerging technologies for the detection of respiratory pathogens. Clin Infect Dis 2011;52:26-30.

27. Dhumpa R, Handberg KJ, Jorgensen PH, Yi S, Wolff A. Rapid detection of avian influenza virus in chicken fecal samples by immunomagnetic capture reverse transcriptase-polymerase chain reaction assay. Diagn Microbiol Infect Dis 2011;69:258-65.

28. Sidoti F, Rizzo F, Costa C, Astegiano S, Curtoni A. Development of real-time RT-PCR assays for detection of type an influenza 
virus and for subtyping of avian $\mathrm{H} 5$ and $\mathrm{H} 7$ hemagglutinin subtypes. Mol Biotechnol 2010;44:41.

29. Yang J, Kim JH, Kim Y. Comparison of nine different qualitative HBsAg assay kits. Korean J Lab Med 2010;30:178-84.

30. Kim H, Shin S, Oh EJ, Kahng J, Kim Y. Comparison of the advan sure HBV real-time PCR test with three other HBV DNA quantification assays. Clin Lab Sci 2013;43:230-7.

31. Mühlbacher A, Schennach H, Van Helden J, Hebell T, Pantaleo G. Performance evaluation of a new fourth-generation HIV combination antigen-antibody assay. Immunol Med Microbiol 2013;202:77-86.

32. Saune K, Delaugerre C, Raymond S, Nicot F, Boineau J. Analytical sensitivity of three real-time PCR assays for measuring subtype B HIV-1 RNA. J Clin Virol 2013;57:80-3

33. Lee KY, Kang H, Ryu SH, Lee DS, Lee JH. Bioimaging of nucleolin aptamer-containing 5-(N-benzylcarboxyamide)-2' deoxyuridine more capable of specific binding to targets in cancer cells. J Biomed Biotechnol 2010;2010:505-10.

34. Min K, Song KM, Cho M, Chun YS, Shim YB. Simultaneous electrochemical detection of both PSMA (+) and PSAMA ( prostate cancer cells using an RNA/peptide dual-aptamer probe. Chem Comm 2010;46:5566-8.

35. Pascual L, Cerqueira Coutinho C, Garcia Fernandez A, de Luis B, Bernardes ES. MUC1 aptamer-capped mesoporous silica nanoparticles for controlled drug delivery and radio-imaging applications. Nanomed Nanotechnol 2017;13:2495-505.

36. Mosafer J, Abnous K, Tafaghodi M, Mokhtarzadeh A, Ramezani M. In vitro and in vivo evaluation of anti-nucleolin-targeted magnetic PLGA nanoparticles loaded with doxorubicin as a theranostic agent for enhanced targeted cancer imaging and therapy. Eur J Pharm Biopharm 2017;113:60-74.

37. Mosafer J, Teymouri M, Abnous K, Tafaghodi M, Ramezani M. Study and evaluation of nucleolin-targeted delivery of magnetic PLGA-PEG nanospheres loaded with doxorubicin to C6 glioma cells compared with low nucleolin-expressing L929 cells. Mater Sci Eng 2017;72:123-33.

38. Savla R, Taratula O, Garbuzenko O, Minko T. Tumor targeted quantum dot-mucin 1 aptamer-doxorubicin conjugate for imaging and treatment of cancer. J Controlled Release 2011;153:16-22.

39. Chen D, Li B, Cai S, Wang P, Peng S. Dual targeting luminescent gold nanoclusters for tumor imaging and deep tissue therapy. Biomaterials 2016;100:1-6.

40. Yu MK, Kim D, Lee IH, So JS, Jeong YY. Image-guided prostate cancer therapy using aptfametionalized thermally cross-linked superparamagnetic iron oxide nanoparticles. Small 2011;7:2241-9.

41. Jalalian SH, Taghdisi SM, Hamedani NS, Kalat SA, Lavaee P. Epirubicin loaded superparamagnetic iron oxide nanoparticleaptamer bioconjugate for combined colon cancer therapy and imaging in vivo. Eur J Pharm Sci 2013;50:191-7.

42. Lei Y, Tang J, Shi H, Ye X, He X. Nature-inspired smart DNA nanodoctor for activatable in vivo cancer imaging and in situ drug release based on the recognition-triggered assembly of split aptamer. Anal Chem 2016;88:11699-706.

43. Röthlisberger P, Gasse C, Hollenstein M. Nucleic acid aptamers emerging applications in medical imaging, nanotechnology, neurosciences, and drug delivery. Int J Mol Sci 2017;18:2430.

44. Tao W, Zeng X, Wu J, Zhu X, Yu X. Polydopamine-based surface modification of novel nanoparticle-aptamer bioconjugates for in vivo breast cancer targeting and enhanced therapeutic effects. Theranostics 2016;6:470.

45. Luo Z, Yan Z, Jin K, Pang Q, Jiang T. Precise glioblastoma targeting by AS1411 aptamer-functionalized poly (l- $\gamma$ glutamylglutamine)-paclitaxel nanoconjugates. J Colloid Interface Sci 2017;490:783-96.

46. Gao H, Qian J, Cao S, Yang Z, Pang Z. Precise glioma targeting of and penetration by aptamer and peptide dual-functioned nanoparticles. Biomaterials 2012;33:5115-23.

47. Gao H, Qian J, Yang Z, Pang Z, Xi Z. Whole-cell SELEX aptamerfunctionalized poly (ethylene glycol)-poly ( $\varepsilon$-caprolactone) nanoparticles for enhanced targeted glioblastoma therapy. Biomaterials 2012;33:6264-72.

48. Karam FF, Alzayd AA. Swelling behavior Of Poly (AAM_MA) hydrogel matrix and study effects $\mathrm{pH}$ and ionic strength, enforcement in controlled release system. Int J Appl Pharm 2018;10:318-25.

49. Soontornworajit B, Zhou J, Shaw MT, Fan TH, Wang Y. Hydrogel functionalization with DNA aptamers for sustained PDGF-BB release. Chem Comm 2010;46:1857-9.

50. Soontornworajit B, Zhou J, Wang Y. A hybrid particle-hydrogel composite for oligonucleotide-mediated pulsatile protein release. Soft Matter 2010;6:4255-61.

51. Soontornworajit B, Zhou J, Snipes MP, Battig MR, Wang Y. Affinity hydrogels for controlled protein release using nucleic acid aptamers and complementary oligonucleotides. Biomaterials 2011;3228:6839-49.

52. Zhang Z, Chen N, Li S, Battig MR, Wang Y. Programmable hydrogels for controlled cell catch and release using hybridized aptamers and complementary sequences. J Am Chem Soc 2012;134:15716-9.

53. Chen N, Zhang Z, Soontornworajit B, Zhou J, Wang Y. Cell adhesion on an artificial extracellular matrix using aptamerfunctionalized PEG hydrogels. Biomaterials 2012;33:1353-62.

54. Kawadkar JI, Chauhan MK, Maharana MA. Nanobiotechnology: application of nanotechnology in diagnosis, drug discovery and drug development. Asian J Pharm Clin Res 2011;4:23-5.

55. Shah A, Parekh P, Parvez Azmi VR, Konale A, Palshikar G. Stem cell: a review. Asian J Pharm Clin Res 2011;4:7-12.

56. Li CJ, Cheng P, Liang MK, Chen YS, Lu Q. MicroRNA-188 regulates an age-related switch between osteoblast and adipocyte differentiation. J Clin Invest 2015;125:1509-22.

57. Ardjomandi N, Niederlaender J, Aicher WK, Reinert $\mathrm{S}$, Schweizer E. Identification of an aptamer binding to human osteogenic-induced progenitor cells. Nucleic Acid Ther 2013;23:44-61. 